\title{
Multiple Relaxation Time Lattice Boltzmann Simulation of 2D Natural Convection in a Square Cavity at High Rayleigh Numbers
}

\author{
Mohamed Mahdi Tekitek* \\ Mathematics Department, Faculty of Science of Tunis, University Tunis El Manar, 2029, \\ Tunis, Tunisia
}

Received 24 November 2015, Accepted (in revised version) 8 May 2017

\begin{abstract}
Natural convection in a square cavity at high Rayleigh numbers is simulated by multiple relaxation time (MRT) lattice Boltzmann method (LBM) with a separate distribution function to solve the temperature. The Rayleigh numbers examined here range from $R a=10^{3}$ to $R a=10^{8}$. For Rayleigh numbers below $10^{8}$, the flow remains stationary and transition occurs beyond $R a=2 \times 10^{8}$. Unsteady results at higher Rayleigh numbers $\left(R a=10^{9}\right.$ and $\left.R a=10^{10}\right)$ are also investigated. To the best of our knowledge, this is the first accurate study which involves the high Rayleigh numbers $R a=10^{9}, 10^{10}$.
\end{abstract}

AMS subject classifications: 65Z05, 76N15, 80A20, 82C20

Key words: Thermal lattice Boltzmann model, multiple relaxation time model, double population, natural convection, square cavity.

\section{Introduction}

Lattice Boltzmann method (LBM) [1,2] has been successfully applied to various hydrodynamic problems and the major advantage of the LBM is its explicit formulation. However, its application to non-isothermal problem is limited because of the numerical instability for thermal models [3]. In general, there are three thermal lattice Boltzmann methods (TLBM) named the multispeed approach [4], the passive scalar approach and the double population approach. The multispeed approach adopts a single distribution function in order to obtain the macroscopic dynamic and thermal equations [4]. However, this approach suffers from lack of numerical stability. The passive scalar approach also called hybrid method consists of approximating the velocity field using LBM and the macroscopic temperature employing different numerical methods (e.g., finite difference or finite volume) $[5,6]$. This approach is more stable than the multispeed approach. It has,

*Corresponding author.

Email: mahdi.tekitek@fst.utm.tn (M. M. Tekitek) 
however, two disadvantages. First, the viscous heat dissipation and compression work done by the pressure can not be incorporated, and second the simplicity of the LBM is lost. The double population method, was first used by He et al. [7]. This approach can be regarded as another version of the passive scalar method. In fact to solve for the macroscopic temperature another LBM distribution is used. This model has a better numerical stability than the multispeed approach, and the viscous heat dissipation and compression work done by the pressure can be solved implicitly. Peng et al. [8] proposed a simplified thermal energy distribution model where the compression work done by the pressure and the viscous heat dissipation are neglected. By introducing a forcing function, Guo et al. [9] proposed a thermal lattice BGK equation with viscous heat dissipation in the incompressible limit. The thermally driven cavity with adiabatic top and bottom walls (also called natural convection in a square cavity) is a classical benchmark to examine the accuracy of the scheme. The solution is given for 4 values of the Rayleigh number $(R a)$, $\left(R a=10^{3}, 10^{4}, 10^{5}\right.$ and $\left.10^{6}\right)$. The value of the Prandtl number $(\mathrm{Pr})$ is equal to 0.71 , which corresponds to a cavity filled by air. The reference solution of this problem is given by De Vahl Davis [10]. To validate the double population LBM method a few researchers [11-15] have carried out the above problem. We note here that most of these works use simple relaxation time (SRT), also called Lattice Boltzmann Bhatngar-Gross-Krook (LBGK). This is due to the extreme simplicity of this method.

In this paper we present a novel double population approach using multiple relaxation time lattice Boltzmann method (MRT-LBM) with D2Q9 (i.e., the notation "DdQ $q$ " denotes a lattice Boltzmann scheme with $d$ space dimensions and $q$ velocities) lattice model for solving velocity field and another D2Q9 for solving macroscopic temperature. First we validate our model by considering natural convection in a square cavity when the flow is laminar (i.e., Rayleigh number is less then $10^{8}$ ). Then, we consider the cases $R a=10^{9}$ and $R a=10^{10}$ where the flow is fully turbulent by using very fine mesh. We show that our result is accurate and the closest to the benchmark result of Le Quéré [16] than previous results using the double population LBM approach [12,13] and [17]. This paper is organized as follows. In Section 2, a brief overview of the MRT D2Q9 for the advection-diffusion problems and the MRT D2Q9 for fluid is presented. In Section 3, the thermal LBM for the simulation of a Boussinesq fluid in a square cavity is introduced. In Section 4, the results of this articles are presented and discussed. Finally, Section 5 concludes the paper.

\section{Multi relaxation time Lattice Boltzmann method}

\subsection{Dynamic field}

We consider the classical model D2Q9 with nine discrete velocities and with three conservations to model fluid problems. Let $\mathcal{L}$ be a regular lattice parametrized by a space step $\Delta x$, composed by a set $\mathcal{L}^{0} \equiv\left\{x_{j} \in(\Delta x \mathbb{Z})\right\}$ of nodes or vertices. $\Delta t$ is the time step of the evolution of LBE and $\lambda \equiv \frac{\Delta x}{\Delta t}$ is a (constant) ve- 
locity ratio between space step and time step. We choose the velocities $v_{i}, i \in$ $(0, \cdots, 8)$, such that $v_{i} \equiv c_{i} \frac{\Delta x}{\Delta t}=c_{i} \lambda$, where the family of vectors $\left\{c_{i}\right\}$ is defined by: $c=(0,0),(1,0),(0,1),(-1,0),(0,-1),(1,1),(-1,1),(-1,-1),(1,-1)$. The LBE scheme is a mesoscopic method which deals with a small number of functions $\left\{f_{i}\right\}$ that can be interpreted as populations of fictitious "particles". The populations $f_{i}$ evolve according to the LBE scheme which can be written as follows [18]:

$$
f_{i}\left(x_{j}, t+\Delta t\right)=f_{i}^{*}\left(x_{j}-v_{i} \Delta t, t\right), \quad 0 \leq i \leq 8,
$$

where the superscript $*$ denotes post-collision quantities. Therefore, during each time increment $\Delta t$ there are two fundamental steps: streaming and collision.

- The streaming step describes the motion of a particle which collided in node $x_{j}-$ $v_{i} \Delta t$ having the velocity $v_{j}$ and goes to the $j^{t h}$ neighboring node $x_{j}$.

- Following d'Humières [19], the collision step is defined in the space of moments. The 9 moments $\left\{m_{\ell}\right\}$ are obtained by a linear transformation of vectors $f_{j}$ :

$$
m_{\ell}=\sum_{j} M_{\ell, j} f_{j}
$$

where the coefficients $M_{k, j}$ of the moments matrix $M$ is given by

$$
M=\left(\begin{array}{ccccccccc}
1 & 1 & 1 & 1 & 1 & 1 & 1 & 1 & 1 \\
0 & \lambda & 0 & -\lambda & 0 & \lambda & -\lambda & -\lambda & \lambda \\
0 & 0 & \lambda & 0 & -\lambda & \lambda & \lambda & -\lambda & -\lambda \\
-4 & -1 & -1 & -1 & -1 & 2 & 2 & 2 & 2 \\
4 & -2 & -2 & -2 & -2 & 1 & 1 & 1 & 1 \\
0 & -2 & 0 & 2 & 0 & 1 & -1 & -1 & 1 \\
0 & 0 & -2 & 0 & 2 & 1 & -1 & -1 & 1 \\
0 & 1 & -1 & 1 & -1 & 0 & 0 & 0 & 0 \\
0 & 0 & 0 & 0 & 0 & 1 & -1 & 1 & -1
\end{array}\right) .
$$

The moments have an explicit physical meaning (see e.g., [20]): $m_{0}=\rho$ is the density, $m_{1}=j_{x}$ and $m_{2}=j_{y}$ are $x$-momentum, $y$-momentum, $m_{3}$ is the energy, $m_{4}$ is proportional to energy square, $m_{5}$ and $m_{6}$ are $x$-energy and $y$-energy fluxes and $m_{7}, m_{8}$ are diagonal and off-diagonal stresses.

To obtain the Navier-Stokes equations as equivalent equations we conserve three moments: $m_{0}=\rho$ density, $m_{1}=j_{x}$ and $m_{2}=j_{y}$ the $x$-momentum and $y$-momentum. The other quantities (non-conserved moments) are assumed to relax towards equilibrium values $\left(m_{\ell}^{e q}\right)$ that are nonlinear functions of the conserved quantities and we set:

$$
m_{\ell}^{*}=\left(1-s_{\ell}\right) m_{\ell}+s_{\ell} m_{\ell}^{e q}, \quad 3 \leq \ell \leq 8,
$$


where $s_{\ell}\left(0<s_{\ell}<2\right.$, for $\left.\ell \in\{3,4, \cdots, 8\}\right)$ are relaxation rates, not necessarily equal to a single value as in the so-called BGK case [21]. The equilibrium values $m_{i}^{e q}$ of the non conserved moments in Eq. (2.4) determine the macroscopic behavior of the scheme (i.e., Eq. (2.1)). Indeed, with the following choice of the equilibrium values:

$$
\begin{cases}m_{3}^{e q}=\alpha \rho+\frac{3}{\rho}\left(j_{x}^{2}+j_{y}^{2}\right), & m_{4}^{e q}=\beta \rho-\frac{3}{\rho}\left(j_{x}^{2}+j_{y}^{2}\right), \\ m_{5}^{e q}=-\frac{j_{x}}{\lambda}, & m_{6}^{e q}=-\frac{j_{y}}{\lambda}, \\ m_{7}^{e q}=\frac{j_{x}^{2}-j_{y}^{2}}{\rho}, & m_{8}^{e q}=\frac{j_{x} j_{y}}{\rho},\end{cases}
$$

and using Taylor expansion (taking $\alpha=-2$ and $s_{7}=s_{8}$ ), we find the following macroscopic equations [18] up to order three in $\Delta t$ :

$$
\begin{aligned}
& \frac{\partial \rho}{\partial t}+\frac{\partial j_{x}}{\partial x}+\frac{\partial j_{y}}{\partial y}=\mathcal{O}\left(\Delta t^{2}\right), \\
& \frac{\partial j_{x}}{\partial t}+\frac{\lambda^{2}}{3} \frac{\partial \rho}{\partial x}+j_{x} \frac{\partial j_{x}}{\partial x}+j_{y} \frac{\partial j_{x}}{\partial y} \\
& =\frac{\lambda^{2}}{3} \Delta t\left[\left(\frac{1}{s_{3}}-\frac{1}{2}\right) \frac{\partial}{\partial x}\left(\frac{\partial j_{x}}{\partial x}+\frac{\partial j_{y}}{\partial y}\right)+\left(\frac{1}{s_{7}}-\frac{1}{2}\right) \Delta j_{x}\right]+\mathcal{O}\left(\Delta t^{2}\right), \\
& \frac{\partial j_{y}}{\partial t}+\frac{\lambda^{2}}{3} \frac{\partial \rho}{\partial y}+j_{x} \frac{\partial j_{y}}{\partial x}+j_{y} \frac{\partial j_{y}}{\partial y} \\
& =\frac{\lambda^{2}}{3} \Delta t\left[\left(\frac{1}{s_{3}}-\frac{1}{2}\right) \frac{\partial}{\partial y}\left(\frac{\partial j_{x}}{\partial x}+\frac{\partial j_{y}}{\partial y}\right)+\left(\frac{1}{s_{7}}-\frac{1}{2}\right) \Delta j_{y}\right]+\mathcal{O}\left(\Delta t^{2}\right) .
\end{aligned}
$$

The parameter $\alpha$ is linked to the speed of sound $c_{s}$ which is equal to $\frac{\lambda}{\sqrt{3}}$. The relaxation parameters $s_{3}$ and $s_{8}$ are directly linked to the transport coefficient. In fact, the bulk viscosity $\zeta$ and shear viscosity $v$ are given by:

$$
\zeta=c_{s}^{2} \Delta t\left(\frac{1}{s_{3}}-\frac{1}{2}\right), \quad v=\frac{\lambda^{2} \Delta t}{3}\left(\frac{1}{s_{7}}-\frac{1}{2}\right),
$$

see [20] for the complete derivation of these properties. The coefficient $\beta$ and the relaxation rates $s_{4}, s_{5}$ and $s_{6}$ play no role for the hydrodynamic behavior of the model, however they are relevant for the stability and the accuracy of the boundary conditions [22].

\subsection{Thermal field}

The thermal field is modeled using the passive scalar approach to enhance the numerical stability, where a separate distribution function is used to solve for the temperature distribution $[8,23]$. The D2Q9 model introduced in the above section is considered. Hence, the evolution of the LB scheme is given by:

$$
g_{i}\left(x_{j}, t+\Delta t\right)=g_{i}^{*}\left(x_{j}-v_{i} \Delta t, t\right), \quad 0 \leq i \leq 8 .
$$


We replace here $f_{i}$ by $g_{i}$ in the Eq. (2.1) since $g_{i}$ is now the energy distribution function. We keep the same choice of the matrix of moments (2.3). The nine moments $\left\{\widetilde{m}_{\ell}\right\}$ are obtained also by a linear transformation of vectors $g_{j}$ :

$$
\widetilde{m}_{\ell}=\sum_{j} M_{\ell, j} g_{j}
$$

To simulate advection-diffusion problems, we conserve only one moment $\widetilde{m}_{0}=T$ (density) in the collision step. The non-conserved moments relax towards equilibrium values $\left(\widetilde{m}_{\ell}^{e q}\right)$ as follows:

$$
\widetilde{m}_{\ell}^{*}=\left(1-\sigma_{\ell}\right) \widetilde{m}_{\ell}+\sigma_{\ell} \widetilde{m}_{\ell}^{e q}, \quad 1 \leq \ell \leq 8,
$$

where $\sigma_{\ell}\left(0<\sigma_{\ell}<2\right.$, for $\left.\ell \in\{1,2, \ldots, 8\}\right)$ are relaxation rates. The equilibrium values $\widetilde{m}_{i}^{e q}$ of the non conserved moments [24] are given by:

$$
\begin{cases}\widetilde{m}_{1}^{e q}=\lambda u T, & \widetilde{m}_{2}^{e q}=\lambda v T, \\ \widetilde{m}_{3}^{e q}=\widetilde{\alpha} T+3 T\left(u^{2}+v^{2}\right), & \widetilde{m}_{4}^{e q}=\widetilde{\beta} \rho, \\ \widetilde{m}_{5}^{e q}=u T\left(-1+3\left(u^{2}+v^{2}\right)\right), & \widetilde{m}_{6}^{e q}=v T\left(-1+3\left(u^{2}+v^{2}\right)\right), \\ \widetilde{m}_{7}^{e q}=T\left(u^{2}-v^{2}\right), & \widetilde{m}_{8}^{e q}=T(u v),\end{cases}
$$

where $\mathbf{v} \equiv(u, v)$ is a given field. We set $\sigma \equiv \sigma_{1}=\sigma_{2}$ and use Taylor expansion [25] or Chapman-Enskog procedure [26] we find the advection-diffusion equations [27] up to order three in $\Delta t$ :

$$
\frac{\partial T}{\partial t}+\lambda \mathbf{v} \cdot \nabla T-\kappa \Delta T=\mathcal{O}\left(\Delta t^{2}\right)
$$

where the value of the diffusivity $\kappa$ is:

$$
\kappa=\Delta t \lambda^{2} \frac{\widetilde{\alpha}+4}{6}\left(\frac{1}{\sigma}-\frac{1}{2}\right)
$$

The D2Q9 for thermal problem can model non isotropic diffusion problems [28,29]. When $u=v=0$, Eq. (2.11) reduces to the isotropic diffusion problem.

\subsection{Coupling of dynamic and thermal fields}

With the Boussinesq approximation, the buoyancy term is assumed to depend linearly on the temperature as follows:

$$
F_{y}=\beta g_{y}\left(T-T_{r e f}\right),
$$

where $\beta$ is the thermal expansion coefficient, $g_{y}$ is the acceleration due to gravity, and $T_{r e f}$ is the reference temperature.

To perform the coupling, the buoyancy force $F_{y}$ is added in the moments space before and after the collision process of the LB scheme as described by Eq. (2.1). The procedure goes like this [22]: 
- The $y$ direction momentum $\left(j_{y}\right)$ and energy flux $\left(q_{y}\right)$ are modified by adding half of the external force $F_{y}$, i.e.,

$$
\bar{j}_{y}=j_{y}+\frac{\Delta t}{2} F_{y}, \quad \bar{q}_{y}=q_{y}-\frac{\Delta t}{2 \lambda} F_{y} .
$$

- Compute the equilibrium moments in Eq. (2.5) using $\bar{j}_{y}$ and $\bar{q}_{y}$ to replace $j_{y}$ and $q_{y}$.

- Perform collision in Eq. (2.1) described by the superscript $*$.

- Post collision in $y$ direction momentum and energy flux are modified by adding another half of the external force, i.e.,

$$
\bar{j}_{y}^{*}=j_{y}^{*}+\frac{\Delta t}{2} F_{y}, \quad \bar{q}_{y}^{*}=q_{y}^{*}-\frac{\Delta t}{2 \lambda} F_{y} .
$$

- Perform streaming in Eq. (2.1) using $\bar{j}_{y}^{*}$ and $\bar{q}_{y}^{*}$ to replace $j_{y}^{*}$ and $q_{y}^{*}$.

Remark 2.1. Other forms of forcing term accounted for the discrete effect could also be adopted [9]. It is noted that the compressibility may influence the results, and this can be eliminated by using an incompressible model [30]. However, since the present Mach number is low, therefore this influence could be neglected [8].

\subsection{Geometry and boundary conditions}

Natural convection in a square cavity $\Omega=[0, H] \times[0, H]$ (see Fig. 1 ) is considered, where the flow is bounded by a stationary square enclosure with sidewalls maintained at different temperatures and driven by the buoyancy force. For laminar convection in this flow configuration, the viscous heat dissipation is assumed to be negligible. The temperature difference between the walls introduces a temperature gradient in the fluid, and the

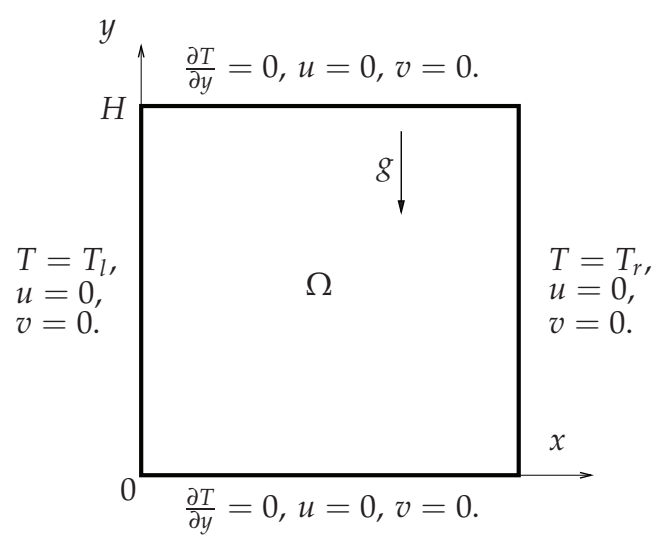

Figure 1: Configuration of natural convection in a square cavity. 


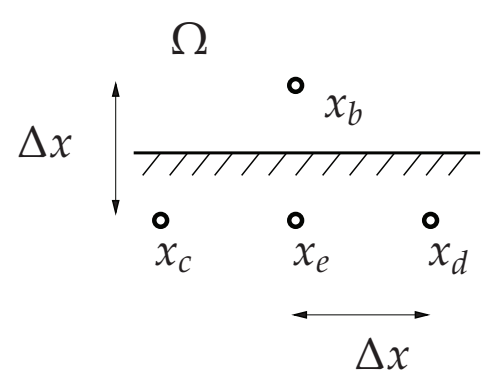

(a)

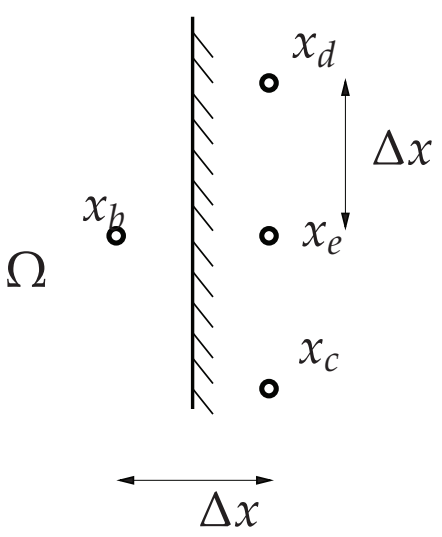

(b)

Figure 2: (a) Boundary node $x_{b}$ in the bottom of the domain $\Omega$. (b) Boundary node $x_{b}$ in the right of the domain $\Omega$.

consequent density difference induces a convective fluid motion. The left wall is at the higher uniform temperature $T_{l}$ and the right wall is at the lower uniform temperature $T_{r}$. Both top and bottom walls are adiabatic, i.e., $\partial T / \partial y=0$. The summary of the boundary conditions is shown below:

$$
\begin{array}{ll}
u=v=0 & \text { on } \partial \Omega, \\
T=T_{l} & \text { on }\{0\} \times[0, H], \\
T=T_{r} & \text { on }\{H\} \times[0, H] \\
\frac{\partial T}{\partial y}=0 & \text { on }[0, H] \times\{0\} \text { and }[0, H] \times\{H\} .
\end{array}
$$

For the Dirichlet boundary condition for the velocity (2.13a) at the walls of the cavity, the classical half way bounce-back boundary condition is adopted. So, for example, consider the bottom wall for a boundary node $x_{b}$ (see left of Fig. 2), the following bounce-back boundary condition is applied:

$$
\left\{\begin{array}{l}
f_{2}\left(x_{b}, t+\Delta t\right)=f_{4}\left(x_{e}, t+\Delta t\right)=f_{4}^{*}\left(x_{b}, t\right), \\
f_{5}\left(x_{b}, t+\Delta t\right)=f_{7}\left(x_{c}, t+\Delta t\right)=f_{7}^{*}\left(x_{b}, t\right), \\
f_{6}\left(x_{b}, t+\Delta t\right)=f_{8}\left(x_{d}, t+\Delta t\right)=f_{8}^{*}\left(x_{b}, t\right) .
\end{array}\right.
$$

For the thermal boundary condition, the Dirichlet boundary conditions given by Eqs. (2.13c) and (2.13b) on the left and right wall of the domain $\Omega$ are introduced. For a given constant temperature $\theta$, this can be archived using the following scheme in bound- 
ary node $x_{b}$ on the right wall (see right of Fig. 2):

$$
\left\{\begin{array}{l}
g_{3}\left(x_{b}, t+\Delta t\right)=-g_{1}\left(x_{e}, t+\Delta t\right)+\frac{1}{36}(4-\widetilde{\alpha}-2 \widetilde{\beta}) \theta \\
g_{7}\left(x_{b}, t+\Delta t\right)=-g_{5}\left(x_{c}, t+\Delta t\right)+\frac{1}{36}(4+2 \widetilde{\alpha}+\widetilde{\beta}) \theta \\
g_{6}\left(x_{b}, t+\Delta t\right)=-g_{8}\left(x_{d}, t+\Delta t\right)+\frac{1}{36}(4+2 \widetilde{\alpha}+\widetilde{\beta}) \theta .
\end{array}\right.
$$

For the Neumann boundary condition on the top and bottom walls of the domain $\Omega$ given by Eq. (2.13d), the classical "bounce back" scheme is adopted. Consider a boundary node $x_{b}$ in the bottom wall (see right of Fig. 2), the following scheme is used:

$$
\left\{\begin{array}{l}
g_{2}\left(x_{b}, t+\Delta t\right)=g_{4}\left(x_{e}, t+\Delta t\right)=g_{4}^{*}\left(x_{b}, t\right), \\
g_{5}\left(x_{b}, t+\Delta t\right)=g_{7}\left(x_{c}, t+\Delta t\right)=g_{7}^{*}\left(x_{b}, t\right), \\
g_{6}\left(x_{b}, t+\Delta t\right)=g_{8}\left(x_{d}, t+\Delta t\right)=g_{8}^{*}\left(x_{b}, t\right) .
\end{array}\right.
$$

For more detail about how to reconstruct the above boundary condition for thermal problem see [28]. In the present parallel implementation, the single program multiple data (SPMD) environment is employed. Message-Passing-Interface (MPI) is adopted for the communication between the processors. The domain decomposition is done in direction of the computational domain, where the ghost cells are adopted along the inter-processor boundary.

\section{Numerical results and discussion}

For the present natural convection within the square cavity as shown in Fig. 1, the major control parameter is the Rayleigh number $R a=\beta \mathbf{g} \Delta T H^{3} \mathrm{Pr} / v^{2}$ associated with the heat transfer within the fluid, where $H$ is either the height or the width of the cavity. The Nusselt number is also an important dimensionless parameter in describing the convective heat transport. Its average in the whole flow domain is defined as,

$$
\overline{N u}=\frac{1}{\chi \Delta T} \int_{0}^{H} q_{x}(x, y) d y,
$$

where $\chi$ is the diffusivity and $q_{x}(x, y)=u T(x, y)-\chi \partial T(x, y) / \partial x$ is the local heat flux in the horizontal direction. To compare with previous results, the main quantities to compute are: $u_{\max }, y, v_{\max }, x$ and $\overline{N u}$, where $u_{\max }$ (resp. $v_{\max }$ ) is the maximal vertical (resp. horizontal) velocity on the horizontal (resp. vertical) midplane of the cavity and its location $y$ (resp. $x$ ) and $\overline{N u}$ is the average Nusselt number. We compute, for some cases, the maximum stream function $\psi_{\max }$ on the whole domain, where the stream function is determined from :

$$
\nabla \cdot(\nabla \times \psi)=\frac{\partial v}{\partial x}-\frac{\partial u}{\partial y}
$$


Note here that for the computation of $\overline{N u}$, described by Eq. (3.1), the temperature gradient $\frac{\partial T}{\partial x}$ is needed. To calculate this gradient we do not perform any additional interpolation. In fact this quantity can be evaluated by using Taylor expansion [28] up to second-order of the non-conserved moment $\widetilde{m}_{1}$ :

$$
\widetilde{m}_{1}=-\lambda^{2} \Delta t \frac{1}{\sigma_{\mathcal{K}}}\left[\frac{4+\widetilde{\alpha}}{6} \frac{\partial T}{\partial x}\right]+\mathcal{O}\left(\Delta t^{2}\right) .
$$

All the velocities are normalized using the diffusion velocity $\chi / H$. The temperature is dimensionless, locations $x$ and $y$ are normalized using $H$.

\subsection{Stability and admissible grid}

Let define the Mach number as follows:

$$
M a=\frac{U}{C_{s}}
$$

where the quantity $U=\sqrt{\alpha g \Delta T H}=\sqrt{\frac{R a}{P r}} \frac{v}{H}$ is the characteristic velocity in thermal convective flows. So, to keep stability of the LBM scheme related to dynamic field, the Mach number should be smaller than the critical value $\frac{3}{10}$ (see [31] for more details). In numerical simulation the parameters are fixed as $P r=0.71$ and $v=0.01$. Then, the Eq. (3.2) gives a constraint on the mesh size $H$ which must verify

$$
\frac{10}{3} \frac{v}{C_{s}} \sqrt{\frac{R a}{P r}}<H
$$

For example, for $R a=10^{7}, R a=10^{8}, R a=10^{9}$ and $R a=10^{10}$ the mesh size must satisfy $H>216, H>685, H>2166$ and $H>6800$, respectively.

\subsection{Grid dependence}

We begin by the study of the grid dependence and the accuracy of the scheme. In fact, Table 1 gives the results for the simulation for Rayleigh number $R a=10^{6}$ by using some different mesh sizes. We note that the calculated values approach the values given by the benchmark of de Vahl Davis [10] and the benchmark of Le Quéré [16]. Moreover, Table 1 shows also second order accuracy for all measured quantities. Here the accuracy is calculated by least squares method using relative error between the solution obtained by $N^{2}$ mesh size, and the reference one obtained by fine mesh $\left(N_{r e f}^{2}=1001^{2}\right)$.

We have also seen the grid dependance in the case of $R a=10^{8}$ for the following mesh sizes: $495 \times 495,987 \times 987$ and $2016 \times 2016$. Table 2 shows that the calculated quantities quickly approach the values given by the benchmark of Le Quéré [16]. We note here that the first grid size does not resolve the stability condition in Eq. (3.3). But this mesh size still gives a good solution. 
Table 1: Grid dependence and order of accuracy for numerical results of simulating 2-D natural convection of air in a square cavity for the case $R a=10^{6}$.

\begin{tabular}{||c|c|c|c|c|c||}
\hline Mesh size & $\overline{N u}$ & $u_{\max }$ & $y$ & $v_{\max }$ & $x$ \\
\hline $47^{2}$ & 9.5729 & 64.6766 & 0.8414 & 222.8704 & $3.6585 \times 10^{-2}$ \\
\hline $57^{2}$ & 9.2079 & 64.8453 & 0.8508 & 218.5256 & $4.3859 \times 10^{-2}$ \\
\hline $81^{2}$ & 8.8913 & 64.8181 & 0.8456 & 218.6303 & $4.3209 \times 10^{-2}$ \\
\hline $161^{2}$ & 8.7828 & 64.8375 & 0.8478 & 220.1784 & $4.0372 \times 10^{-2}$ \\
\hline $225^{2}$ & 8.8006 & 64.8393 & 0.8511 & 220.7288 & $3.7777 \times 10^{-2}$ \\
\hline $321^{2}$ & 8.8139 & 64.8403 & 0.8489 & 220.5115 & $3.8940 \times 10^{-2}$ \\
\hline $615^{2}$ & 8.8226 & 64.8391 & 0.8495 & 220.5741 & $3.8211 \times 10^{-2}$ \\
\hline Reference solution & 8.8241 & 64.8372 & 0.8495 & 220.5739 & $3.7993 \times 10^{-2}$ \\
\hline Accuracy & 2.109 & 2.0154 & 1.9261 & 2.0592 & 1.7571 \\
\hline Davis [10] & 8.817 & 64.630 & 0.850 & 219.360 & $3.8 \times 10^{-2}$ \\
\hline Le Quéré [16] & 8.825 & 64.483 & 0.850 & 220.559 & $3.8 \times 10^{-2}$ \\
\hline
\end{tabular}

Table 2: Grid dependence and order of accuracy for numerical results of simulating 2-D natural convection of air in a square cavity for the case $R a=10^{8}$.

\begin{tabular}{||c|c|c|c|c|c||}
\hline Mesh size & $\mathrm{Nu}$ & $\mathrm{Umax}$ & $\mathrm{Y}$ & $\mathrm{Vmax}$ & $\mathrm{X}$ \\
\hline $495^{2}$ & 29.97 & 313.97 & 0.926 & 2219.3 & 0.011 \\
\hline $987^{2}$ & 30.20 & 319.81 & 0.927 & 2222.8 & 0.011 \\
\hline $2016^{2}$ & 30.22 & 321.59 & 0.928 & 2222.7 & 0.012 \\
\hline Le Quéré [16] & 30.22 & 321.88 & 0.928 & 2222.2 & 0.012 \\
\hline
\end{tabular}

\subsection{First test case $\left(10^{3} \leq R a \leq 10^{6}\right)$}

Many papers study the square heated cavity for the following 4 values of the $R a$ numbers: $R a=10^{3}, 10^{4}, 10^{5}$ and $10^{6}$. So we compare our results to the following results: de Vahl Davis [10] benchmark solution where second order finite difference scheme and a Richardson extrapolation scheme are used, Mayne et al. [32] who used h-adaptive finite elements method, Kuznik et al. [13] Dixit et al. [12] and Liu et al. [33] who performed TLBM based on the BGK and Mezrhab et al. [17], Wang et al. [31] who used TLBM based on MRT D2Q9 for the flow and MRT D2Q5 for the temperature. Table 3 shows the numerical results where the domain is covered by a lattice of size $105 \times 105,155 \times 155,205 \times 205$ and $255 \times 255$, respectively for $R a=10^{3}, 10^{4}, 10^{5}$ and $10^{6}$ compared to the results obtained by the methods listed above.

The simulated results are contrasted with the benchmark solutions of De Vahl Davis [10] and the agreements are satisfactory. It is also noted that differences of the predicted velocities and average Nusselt number are less than $0.1 \%$.

Figs. 3, 4, 5, and 6 show respectively, the isotherms, streamlines, horizontal velocities, vertical velocities. Note here that the isotherms are calculated at different Rayleigh numbers and predicted by the present double D2Q9 MRT LBE method. 
Table 3: Comparison of predicted numerical results. Davis [10], Mayne et al. [32], Liu et al. [33], Dixit et al. [12], Kuznik et al. [13], Mezrhab et al. [17] and Wang et al. [31].

\begin{tabular}{||c|c|cccccccc||}
\hline$R a$ & & {$[10]$} & {$[32]$} & {$[33]$} & {$[12]$} & {$[13]$} & {$[17]$} & {$[31]$} & Present \\
\hline \multirow{4}{*}{$10^{3}$} & $u_{\max }$ & 3.649 & 3.6493 & 3.649 & 3.6529 & 3.636 & 3.667 & 3.6494 & 3.6494 \\
& $y$ & 0.813 & 0.8125 & 0.810 & 0.8125 & 0.809 & - & 0.8132 & 0.8146 \\
& $v_{\max }$ & 3.697 & 3.6962 & 3.698 & 3.682 & 3.686 & 3.714 & 3.6974 & 3.6975 \\
& $x$ & 0.178 & 0.1790 & 0.180 & 0.17183 & 0.174 & - & 0.1783 & 0.1791 \\
& $N u$ & 1.1178 & 1.1149 & 1.115 & 1.11272 & 1.117 & 1.112 & 1.1178 & 1.1178 \\
\hline \multirow{4}{*}{$10^{4}$} & $u_{\max }$ & 16.178 & 16.1798 & 16.154 & 16.163 & 16.167 & 16.202 & 16.1834 & 16.1855 \\
& $y$ & 0.823 & 0.8235 & 0.820 & 0.828 & 0.821 & - & 0.8232 & 0.8239 \\
& $v_{\max }$ & 19.617 & 19.6177 & 19.614 & 19.569 & 19.597 & 19.644 & 19.6278 & 19.6294 \\
& $x$ & 0.119 & 0.1195 & 0.120 & 0.125 & 0.120 & - & 0.1189 & 0.1199 \\
& $N u$ & 2.243 & 2.2593 & 2.229 & 2.247 & 2.246 & 2.241 & 2.2448 & 2.2444 \\
\hline \multirow{4}{*}{$10^{5}$} & $u_{\max }$ & 34.73 & 34.7741 & 34.508 & 35.521 & 34.962 & 34.805 & 34.7430 & 34.7470 \\
& $y$ & 0.855 & 0.8535 & 0.855 & 0.8554 & 0.854 & - & 0.8546 & 0.8551 \\
& $v_{\max }$ & 68.590 & 68.6920 & 68.595 & 68.655 & 68.578 & 68.630 & 68.6318 & 68.6323 \\
& $x$ & 0.066 & 0.0665 & 0.065 & 0.0664 & 0.067 & - & 0.06588 & 0.066978 \\
& $N u$ & 4.519 & 4.4832 & 4.489 & 4.5226 & 4.518 & 4.519 & 4.5218 & 4.5200 \\
\hline \multirow{1}{*}{$10^{6}$} & $u_{\max }$ & 64.63 & 64.6912 & 63.456 & 64.186 & 64.133 & 64.793 & 64.8277 & 64.8403 \\
& $y$ & 0.850 & 0.8460 & 0.848 & 0.8496 & 0.860 & - & 0.8498 & 0.8489 \\
& $v_{\max }$ & 219.360 & 220.8331 & 219.788 & 219.866 & 220.537 & 219.663 & 220.5506 & 220.5115 \\
& $x$ & 0.0379 & 0.0380 & 0.036 & 0.0371 & 0.038 & - & 0.03779 & 0.03894 \\
& $N u$ & 8.799 & 8.8811 & 8.750 & 8.805 & 8.792 & 8.817 & 8.8192 & 8.8139 \\
\hline
\end{tabular}

\subsection{Comparison of D2Q9 and D2Q5 for solving thermal problem}

As described by Eq. (2.11), we have an isotropic problem for temperature. This allows us to use a D2Q5 scheme (with only 5 discrete velocities : $\left\{c_{0}, c_{1}, \cdots, c_{4}\right\}$ ) to solve the advection-diffusion equation. Table 4 shows the main values obtained by using D2Q9 and D2Q5 compared to De Vahl Davis "benchmark solutions" [10] for different Rayleigh numbers employing different mesh sizes. The numerical results obtained by both methods are very close to each other and to De Vahl Davis "benchmark solutions".

Remark 3.1. Note that using D2Q5 for temperature is faster and requires less memory than using D2Q9. The advantage of using D2Q9 is the ability to model non isotropic thermal problem.

\subsection{Second test case $\left(10^{7} \leq R a \leq 10^{8}\right)$}

One of the main interests of this paper is to give a "benchmark solutions" for the values of the Rayleigh number equal to $10^{7}$ and $10^{8}$. Table 5 shows results obtained for $R a=10^{7}$ and $R a=10^{8}$ using a fine mesh with $987 \times 987$ and $2016 \times 2016$ nodes, respectively.

Table 6 gives comparison between present benchmark solutions and solutions obtained by other methods (see $[12,13,16,17,34]$ and [35]) for $R a=10^{7}$ and $R a=10^{8}$. Note here that the present results and Contrino et al. [35] results are the closest ones to Le Quéré [16] results. 


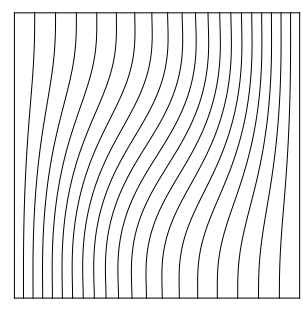

(a) $R a=10^{3}$

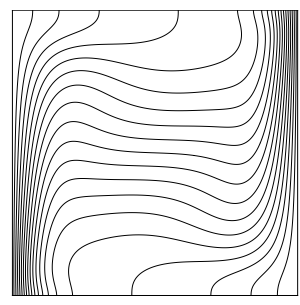

(c) $R a=10^{5}$

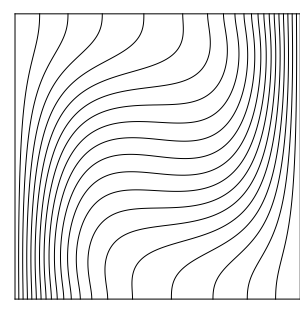

(b) $R a=10^{4}$

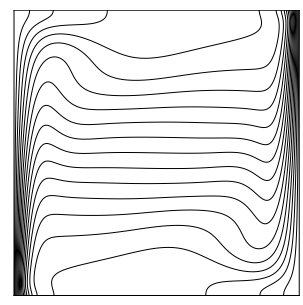

(d) $R a=10^{6}$

Figure 3: The isotherm profiles of 2-D natural convection in a square cavity at different Rayleigh numbers.

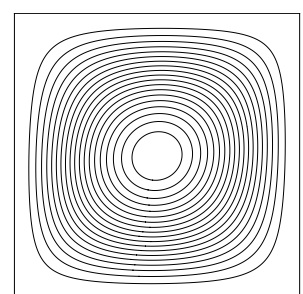

(a) $R a=10^{3}$

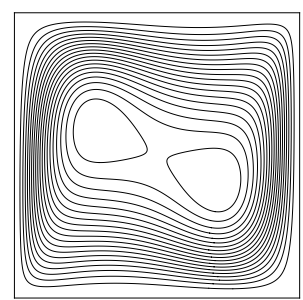

(c) $R a=10^{5}$

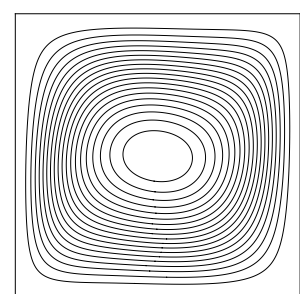

(b) $R a=10^{4}$

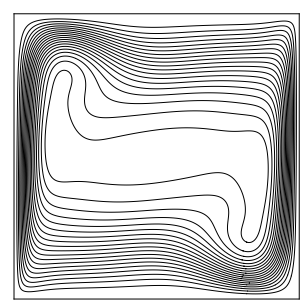

(d) $R a=10^{6}$

Figure 4: The streamline profiles of 2-D natural convection in a square cavity at different Rayleigh numbers. 


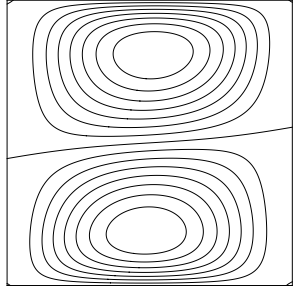

(a) $R a=10^{3}$

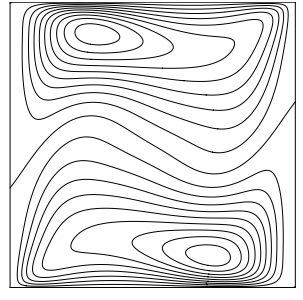

(c) $R a=10^{5}$

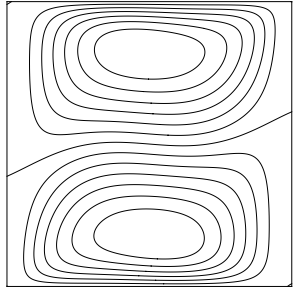

(b) $R a=10^{4}$

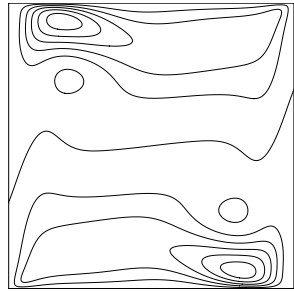

(d) $R a=10^{6}$

Figure 5: The horizontal velocity $u$ profiles of 2-D natural convection in a square cavity at different Rayleigh numbers.

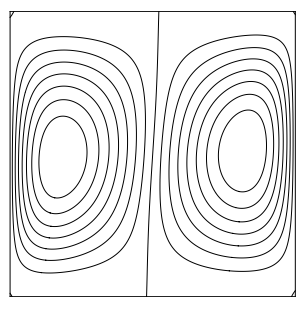

(a) $R a=10^{3}$

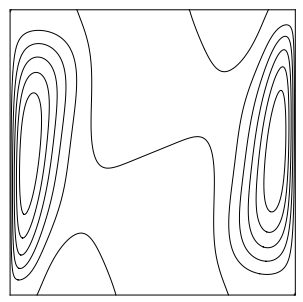

(c) $R a=10^{5}$

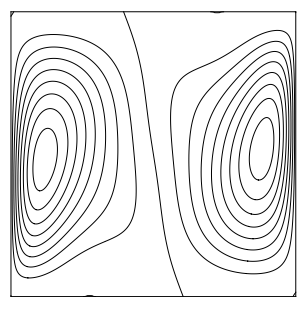

(b) $R a=10^{4}$

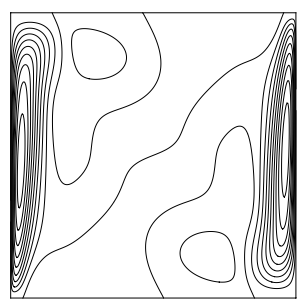

(d) $R a=10^{6}$

Figure 6: The vertical velocity $v$ profiles of 2-D natural convection in a square cavity at different Rayleigh numbers. 
Table 4: Comparison of laminar flow numerical results between using D2Q9 and D2Q5 for solving advection diffusion equation.

\begin{tabular}{|c|c|c|c|c|}
\hline$R a$ & & Davis [10] & D2Q5-D2Q9 & D2Q9-D2Q9 \\
\hline \multirow{6}{*}{$10^{3}$} & $\psi_{\max }$ & 1.174 & 1.1744 & 1.1744 \\
\hline & $u_{\max }$ & 3.649 & 3.6493 & 3.6494 \\
\hline & $y$ & 0.813 & 0.8146 & 0.8146 \\
\hline & $v_{\max }$ & 3.697 & 3.6973 & 3.6975 \\
\hline & $x$ & 0.178 & 0.1791 & 0.1791 \\
\hline & $\overline{N u}$ & 1.1178 & 1.1177 & 1.1178 \\
\hline \multirow{6}{*}{$10^{4}$} & $\psi_{\max }$ & 5.071 & 5.0727 & 5.0734 \\
\hline & $u_{\max }$ & 16.178 & 16.1833 & 16.1855 \\
\hline & $y$ & 0.823 & 0.8239 & 0.8239 \\
\hline & $v_{\max }$ & 19.617 & 19.6272 & 19.6294 \\
\hline & $x$ & 0.119 & 0.1199 & 0.1199 \\
\hline & $\overline{N u}$ & 2.243 & 2.2448 & 2.2444 \\
\hline \multirow{6}{*}{$10^{5}$} & $\psi_{\max }$ & 9.612 & 9.6149 & 9.6157 \\
\hline & $u_{\max }$ & 34.73 & 34.7415 & 34.7470 \\
\hline & $y$ & 0.855 & 0.8551 & 0.8551 \\
\hline & $v_{\max }$ & 68.590 & 68.6260 & 68.6323 \\
\hline & $x$ & 0.066 & 0.066978 & 0.066978 \\
\hline & $\overline{N u}$ & 4.519 & 4.5212 & 4.5200 \\
\hline \multirow{6}{*}{$10^{6}$} & $\psi_{\max }$ & 16.750 & 16.8030 & 16.8039 \\
\hline & $u_{\max }$ & 64.63 & 64.8427 & 64.8403 \\
\hline & $y$ & 0.850 & 0.8489 & 0.8489 \\
\hline & $v_{\max }$ & 219.360 & 220.5248 & 220.5115 \\
\hline & $x$ & 0.0379 & 0.038940 & 0.038940 \\
\hline & $\overline{N u}$ & 8.800 & 8.8194 & 8.8139 \\
\hline
\end{tabular}

Table 5: Benchmark solutions for $R a=10^{7}$ and $R a=10^{8}$ using $987 \times 987$ and $2016 \times 2016$ nodes, respectively.

\begin{tabular}{||ccc||}
\hline$R a$ & $10^{7}$ & $10^{8}$ \\
\hline$\psi_{\max }$ & 30.1564 & 53.8356 \\
\hline$u_{\max }$ & 148.5561 & 321.5927 \\
\hline$y$ & 0.8789 & 0.9278 \\
\hline$v_{\max }$ & 699.1368 & 2222.7265 \\
\hline$x$ & 0.0217 & 0.0121 \\
\hline$\overline{N u}$ & 16.5216 & 30.2218 \\
\hline
\end{tabular}

The solutions corresponding to the values $R a=10^{7}$ and $R a=10^{8}$ are presented in Figs. 7 and 8 in the form of temperature, streamlines and pressure. 
Table 6: Comparison with other works: Le Quéré [16], Markatos et al. [34], Dixit et al. [12], Kuznik et al. [13], Mezrhab et al. [17] and Contrino et al. [35] for $R a=10^{7}, R a=10^{8}, 10^{9}$ and $10^{10}$.

\begin{tabular}{||cccccccc||}
\hline$R a=10^{7}$ & Present & {$[16]$} & {$[34]$} & {$[12]$} & {$[13]$} & {$[17]$} & {$[35]$} \\
\hline$\psi_{\max }$ & 30.1564 & 30.1649 & - & - & - & 30.140 & 30.176 \\
$u_{\max }$ & 148.5561 & 148.5954 & - & 164.236 & 148.768 & 148.400 & 148.585 \\
$y$ & 0.8789 & 0.879 & - & 0.851 & 0.881 & - & 0.8793 \\
$v_{\max }$ & 699.1368 & 699.1795 & - & 701.922 & 702.029 & 998.300 & 699.3224 \\
$x$ & 0.0217 & 0.021 & - & 0.020 & 0.020 & - & 0.0213 \\
$\overline{N u}$ & 16.5216 & 16.523 & - & 16.79 & 16.408 & 16.510 & 16.5230 \\
\hline$R a=10^{8}$ & Present & {$[16]$} & {$[34]$} & {$[12]$} & {$[13]$} & {$[17]$} & {$[35]$} \\
\hline$\psi_{\max }$ & 53.8356 & 53.8475 & - & - & - & 53.323 & 53.9149 \\
$u_{\max }$ & 321.5927 & 321.875 & 514.3 & 389.877 & 321.457 & 305.332 & 321.9063 \\
$y$ & 0.9278 & 0.928 & 0.941 & 0.937 & 0.940 & - & 0.5530 \\
$v_{\max }$ & 2222.7265 & 2222.39 & 1812 & 2241.374 & 2243.36 & 2169.562 & 2222.3279 \\
$x$ & 0.0121 & 0.012 & 0.0135 & 0.0112 & 0.0121 & - & 0.0120 \\
\hline$N u$ & 30.2218 & 30.225 & 32.045 & 30.506 & 29.819 & 30.033 & 30.2241 \\
\hline$R a=10^{9}$ & Present & {$[16]$} & {$[34]$} & {$[12]$} & {$[13]$} & {$[17]$} & {$[35]$} \\
\hline$u_{\max }$ & 705.6806 & - & - & 503.24 & - & - & - \\
$y$ & 0.9765 & - & - & 0.966 & - & - & - \\
$v_{\max }$ & 7175.0103 & - & - & 6820.07 & - & - & - \\
$x$ & 0.0064935 & - & - & 0.0064 & - & - & - \\
\hline$N u$ & 54.5267 & - & - & 57.350 & - & - & - \\
\hline$R a=10^{10}$ & Present & {$[16]$} & {$[34]$} & {$[12]$} & {$[13]$} & {$[17]$} & {$[35]$} \\
\hline$u_{\max }$ & 2441.5322 & - & 2323 & 2323 & - & - & - \\
$y$ & 0.9855 & - & 0.9625 & 0.94023 & - & - & - \\
$v_{\max }$ & 23004.8136 & - & 16890 & 21463 & - & - & - \\
$x$ & $3.9370 .10-3$ & - & 0.0055 & 0.49072 & - & - & - \\
\hline$N u$ & 102.2830 & - & 156.85 & 103.663 & - & - & - \\
\hline
\end{tabular}

\subsection{The unsteady case $\left(R a>10^{8}\right)$}

An approximation of the Rayleigh value when the solution becomes unsteady is given in Paolucci et al. [36] and Le Quéré [16] where they showed that the solution of the 2-D

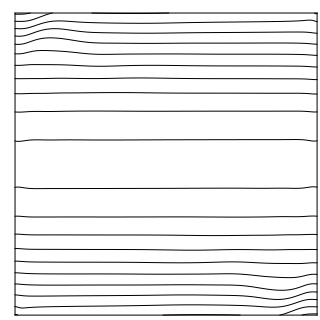

(a)

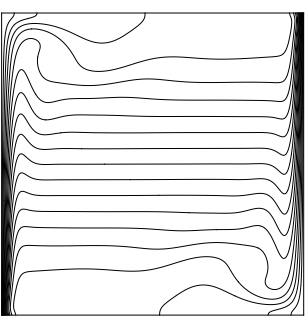

(b)

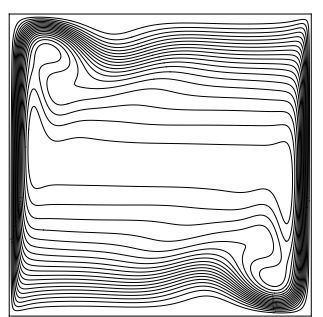

(c)

Figure 7: Pressure field (a), Isotherms (b), Streamlines (c) for $R a=10^{7}$. 


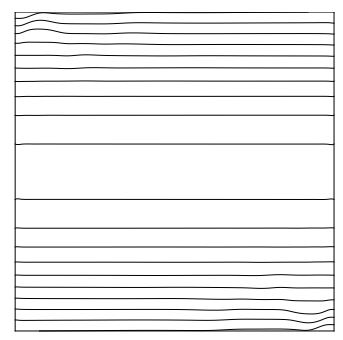

(a)

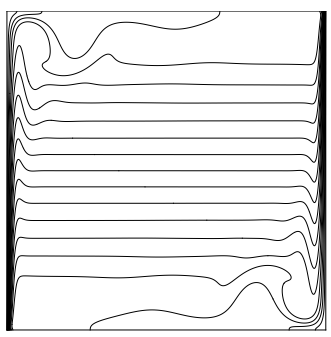

(b)

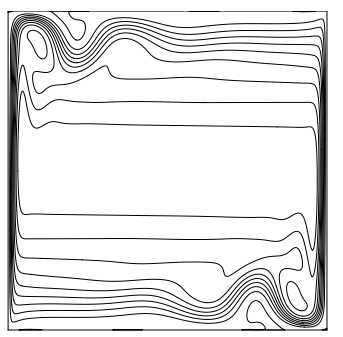

(c)

Figure 8: Pressure field (a), Isotherms (b), Streamlines (c) for $R a=10^{8}$.

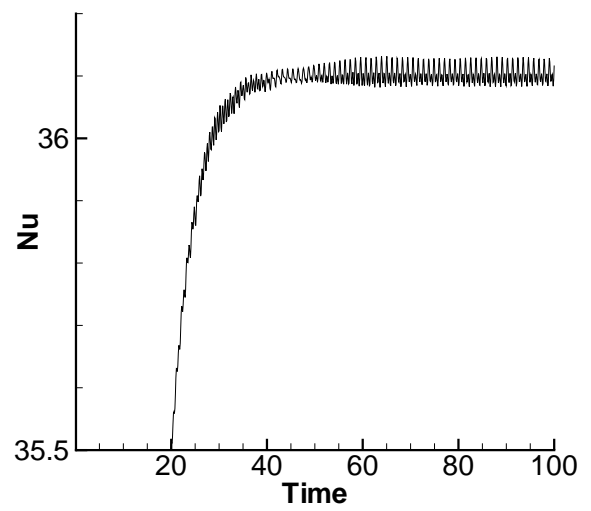

Figure 9: The Nusselt $\overline{N u}$ versus time steps for $R a=2.05 \times 10^{8}$.

natural convection in a square cavity with imposed temperature becomes unsteady for a value of Rayleigh very close to $2 \times 10^{8}$. Our present TLBM gives a steady-state solution for $R a=2 \times 10^{8}$ and an unsteady solution for $R a=2.05 \times 10^{8}$.

Figs. 9 and 10 give the time evolution of the values $\overline{N u}, u_{\max }, y, v_{\max }$, and $x$ for $R a=2.05 \times 10^{8}$. Note here that the solution in this case is periodic. In fact Fig. 11 plots the predicted time-varying Nusselt number $\overline{N u}$ (see Fig. 11, left) and its corresponding power spectrum (see Fig. 11, right).

For the values $R a=10^{9}$ and $R a=10^{10}$ we have unsteady solution, these cases will be the subject of a future work. Figs. 12,13 and 14 give the time evolution of the $\overline{N u}$ Nusselt number $u_{\max }$ and its location $y$, the maximum vertical velocity on the horizontal midplane of the cavity, $v_{\max }$ and its location $x$, the maximum horizontal velocity on the vertical midplane of the solution for Rayleigh number $R a=10^{9}$ and $R a=10^{10}$.

Table 6 compares the average of the above quantities to the results of Markatos et al. [34] and Dixit et al. [12]. Table 7 gives the maximum, minimum and average of the main calculated values $u_{\max }, y, v_{\max }, x$ and $\overline{N u}$. 


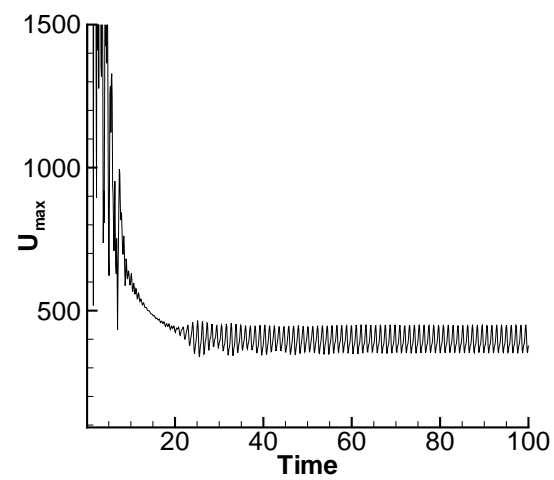

(a) $u_{\max }$

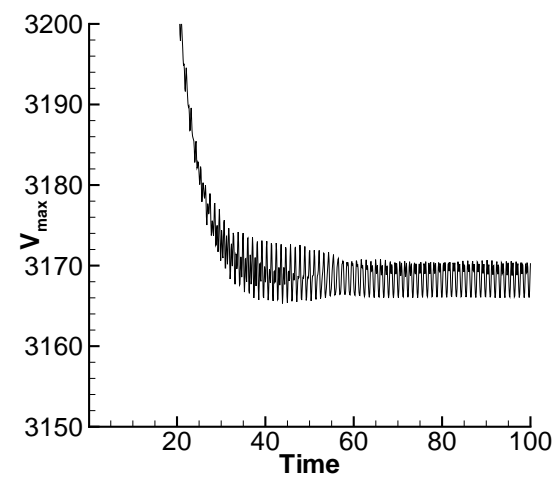

(c) $v_{\max }$

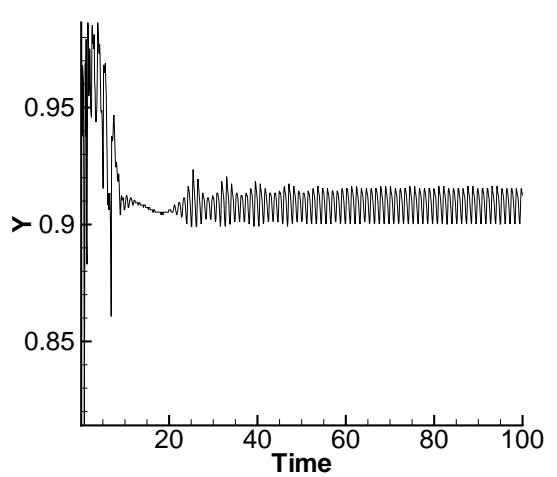

(b) $y$

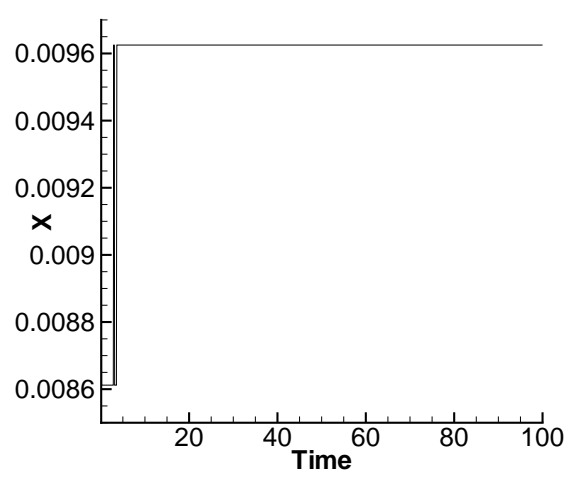

(d) $x$

Figure 10: The values $u_{\max }, y, v_{\max }$ and $x$ versus time steps for $R a=2.05 \times 10^{8}$.
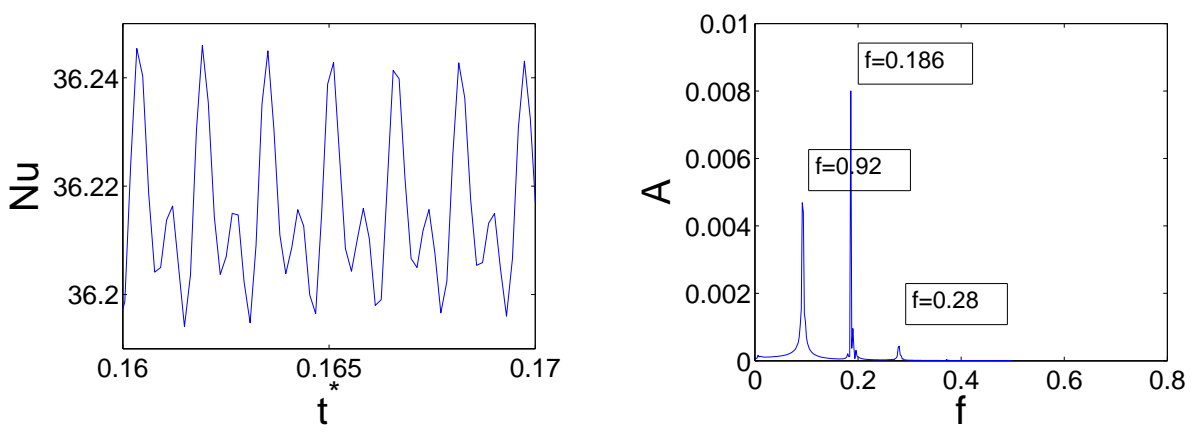

Figure 11: The power spectrum of the Nusselt $\overline{N u}$. (a) Time evolving of the Nusselt; (b) FFT plot.

\section{Conclusions}

In this paper, a multi-relaxation time thermal lattice Boltzmann scheme has been applied to compute natural convection flow within differential heated square cavity. For Rayleigh 


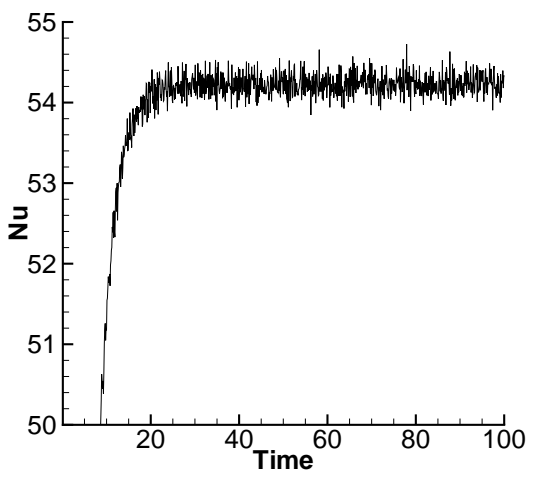

(a) $R a=10^{9}$

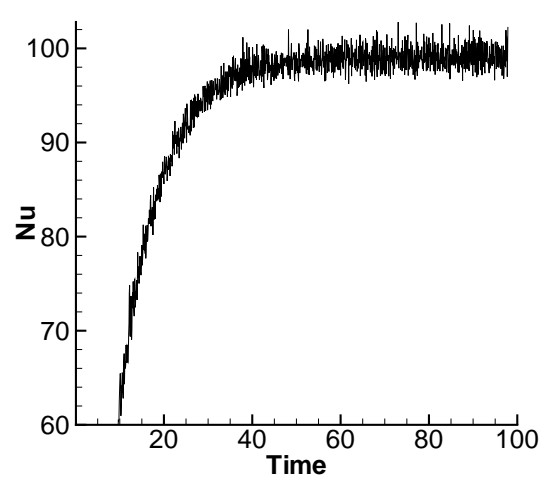

(b) $R a=10^{10}$

Figure 12: The Nusselt $\overline{N u}$ versus time steps (a) for $R a=10^{9}$; (b) for $R a=10^{10}$.

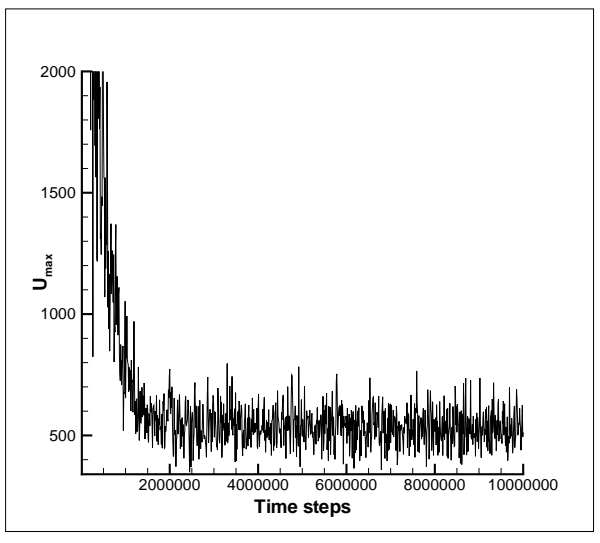

(a) $u_{\max }$

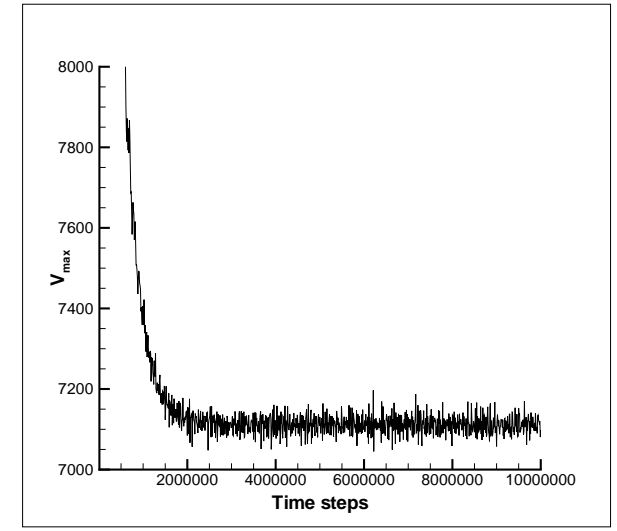

(c) $v_{\max }$

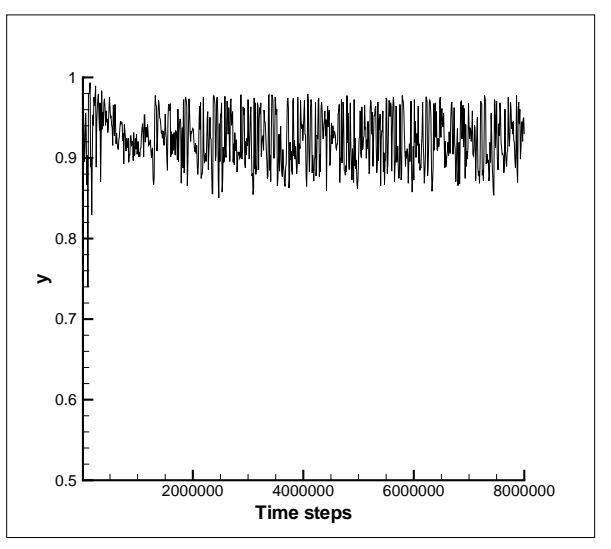

(b) $y$

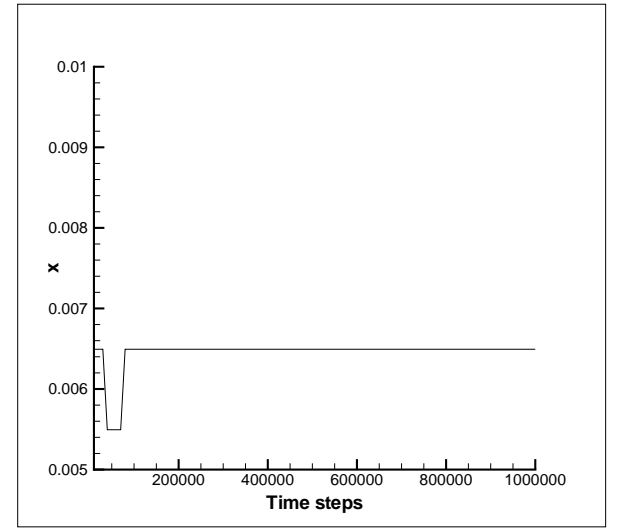

(d) $x$

Figure 13: The values $u_{\max }, y, v_{\max }$ and $x$ versus time steps for $R a=10^{9}$. 


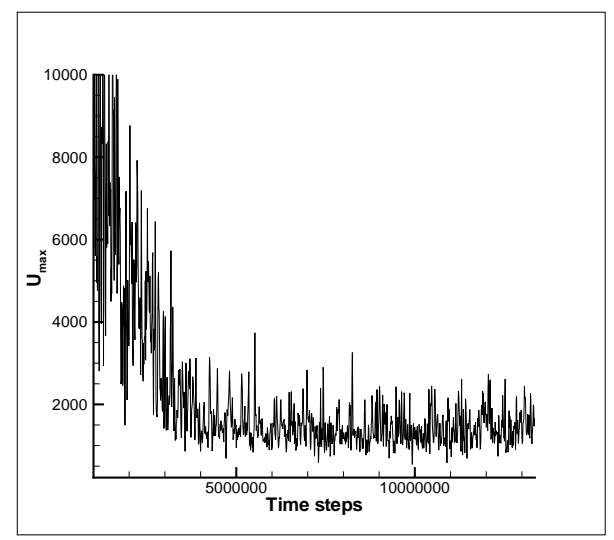

(a) $u_{\max }$

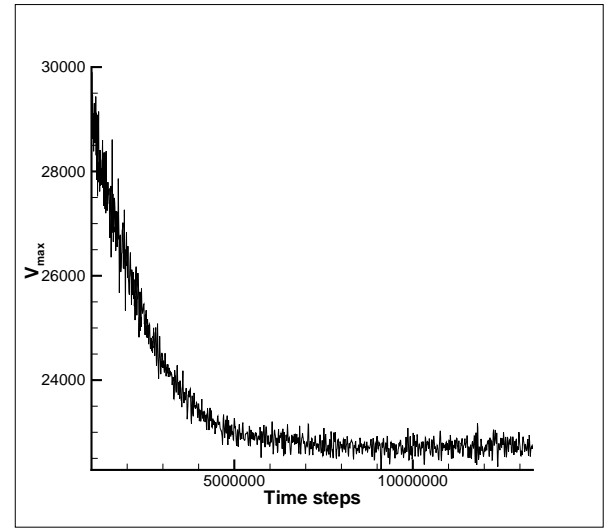

(c) $v_{\max }$

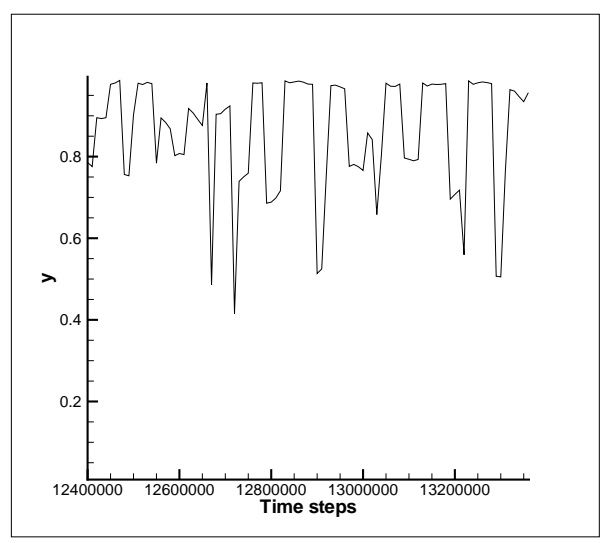

(b) $y$

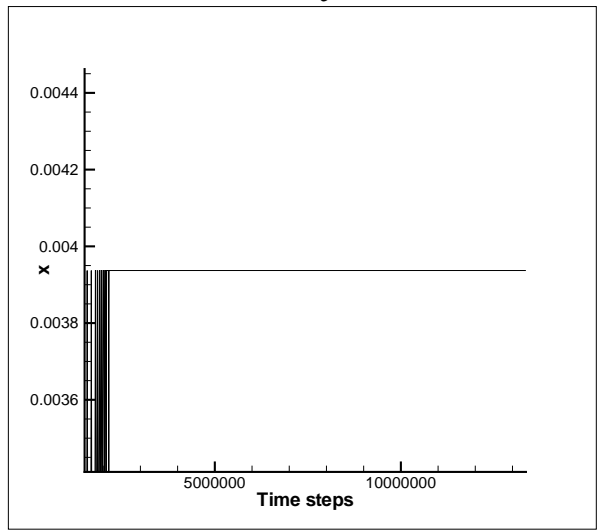

(d) $x$

Figure 14: The values $u_{\max }, y, v_{\max }$ and $x$ versus time steps for $R a=10^{10}$.

Table 7: Numerical results for $R a=10^{9}$ and $R a=10^{10}$.

\begin{tabular}{||cccccc||}
\hline$R a$ & $N^{2}$ & & $\max$ & $\min$ & average \\
\hline & & $\overline{N u}$ & 54.5267 & 53.9497 & 54.2109 \\
10 & & $u_{\max }$ & 705.6806 & 398.5403 & 530.8379 \\
& $1001^{2}$ & $y$ & 0.9765 & 0.8746 & 0.9231 \\
& & $v_{\max }$ & 7175.0103 & 7055.7060 & 7110.7323 \\
& & $x$ & 0.0064935 & 0.0064935 & 0.0064935 \\
\hline \multirow{4}{*}{$10^{10}$} & \multirow{2}{*}{$2001^{2}$} & $\overline{N u}$ & 102.2830 & 96.6169 & 99.1093 \\
& & $u_{\max }$ & 2441.5322 & 897.1037 & 1569.4172 \\
& & $v_{\max }$ & 23004.8136 & 22536.3544 & 22730.24122 \\
& & $x$ & $3.9370 .10^{-3}$ & $3.9370 .10^{-3}$ & $3.9370 .10^{-3}$ \\
\hline
\end{tabular}


number under $10^{6}$ the present results compare favorably with previous benchmark solutions. For $R a=10^{7}$ and $R a=10^{8}$ by using a very fine mesh the present scheme produces the best results by reference to the benchmark solution of Le Quéré [16]. Finally, a higher Rayleigh number was also investigated. In fact the solution becomes unsteady around $R a=2.05 \times 10^{8}$ and a description of the mean values are given for $R a=10^{9}$ and $R a=10^{10}$. The novelty of this work relies in the achievement of an accurate simulation of 2D natural convection in a square cavity at a very high Rayleigh numbers, namely $R a=10^{9}, 10^{10}$.

\section{References}

[1] D. Z. YU, R. W. MEI, L.-S. LUO AND W. SHYY, Viscous flow computations with the method of lattice Boltzmann equation, Progress Aerospace Sci., 39 (2003), pp. 329.

[2] D. Bespalko, A. Pollard AND M. UdDIN, Analysis of the pressure fluctuations from an LBM simulation of turbulent channel flow, Comput. Fluids, 54 (2012), pp. 143.

[3] G. McNamara, A. L. Garcia AND B. J. Alder, Stabilization of thermal lattice Boltzmann models, J. Stat. Phys., 81 (1995), pp. 395.

[4] F. J. Alexander, S. Chen, AND J. D. Sterling, Lattice Boltzmann thermodynamics, Phys. Rev. E, 47 (1993), pp. 2249.

[5] F. Massaioli, R. Benzi And S. SuCCI, Exponential tails in two-dimensional Rayleigh-Bénard convection, Europhys. Lett., 21 (1993), pp. 305.

[6] P. LALlemAND AND L.-S. LUO, Hybrid finite-difference thermal lattice Boltzmann equation, Int. J. Modern Phys. B, 17 (2003), pp. 41.

[7] X. HE, S. CHEN AND G. D. DOOLEN, A novel thermal model for the lattice Boltzmann method in incompressible limit, J. Comput. Phys., 146 (1998), pp. 282.

[8] Y. PENG, C. SHU, AND Y. T. CHEW, Simplified thermal lattice Boltzmann model for incompressible thermal flows, Phys. Rev. E, 68 (2003), 026701.

[9] Z. GuO, C. ZHENG AND B. SHI, Discrete lattice effects on the forcing term in the lattice Boltzmann method, Phys. Rev. E, 65 (2002), 046308.

[10] G. DE VAHL DAVIS, Natural convection of air in a square cavity: a bench mark numerical solution, Int. J. Numer. Methods Fluids, 3(1983), pp. 249.

[11] Z. GUO, B. SHI AND C. ZHENG, A coupled lattice BGK model for the Boussinesq equations, Int. J. Numer. Methods Fluids, 39 (2002), pp. 325.

[12] H. N. DiXIT AND V. BABU, Simulation of high Rayleigh number natural convection in a square cavity using the lattice Boltzmann method, Int. J. Heat Mass Trans., 49 (2006), pp. 727.

[13] F. KUZNIK, J. VAReILles, G. RUSAOUEN AND G. KRAUSS, A double-population lattice Boltzmann method with non-uniform mesh for the simulation of natural convection in a square cavity, Int. J. Heat Fluid Flow, 28 (2007), pp. 862.

[14] Z. GUO, C. ZHENG AND B. SHI, Thermal lattice Boltzmann equation for low Mach number flows: Decoupling model, Phys. Rev. E, 75 (2007), pp. 15.

[15] H.-C. MAI, K.-H. LIN, C.-H. YANG AND C.-A. LIN, A thermal lattice Boltzmann model for flows with viscous heat dissipation, Comput. Modeling Eng. Sci., 61 (2010), pp. 45.

[16] P. LE QUÉRÉ, Accurate solutions to the square differentially heated cavity at high Rayleigh number, Comput. Fluids, 20 (1961), pp. 19.

[17] A. Mezrhab, M. A. Moussaoui, M. JAmi, H. NAJI AND M. Bouzidi, Double MRT thermal lattice Boltzmann method for simulation convective flows, Phys. Lett. A, 374 (2010), pp. 3499. 
[18] F. Dubois, Une introduction au schéma de Boltzmann sur réseau, ESAIM: Proceedings, 18 (2007), pp. 181.

[19] D. D'HUMières, Generalized lattice Boltzmann equations, in Rarefied Gas Dynamics: Theory and Simulations, AIAA, Progress in Astronautics and Aeronautics, 159 (1992), pp. 450.

[20] P. LALlemAND AND L.-S. LUO, Theory of the lattice Boltzmann method: Dispersion, dissipation, isotropy, Galilean invariance, and stability, Phys. Rev. E, 61 (2000), pp. 6546.

[21] Y. H. QIAN, D. D'HuMIÈRES, AND P. LALLEMAND, Lattice BGK model for Navier-Stokes equation, Europhys. Lett., 17 (1992), pp. 479.

[22] F. Dubois, P. Lallemand AND M. M. TeKiTEK, On a superconvergent lattice Boltzmann boundary scheme, Comput. Math. Appl., 59 (2010), pp. 2141.

[23] Y. SHI, T. S. ZHAO, AND Z. L. GUO, Thermal lattice Bhatnagar-Gross-Krook model for flows with viscous heat dissipation in the incompressible limit, Phys. Rev. E, 70 (2004), 066310.

[24] P. Lallemand, Constructing Lattice Boltzmann Models with Increasing Accuracy, Spring School on the Lattice Boltzmann Method, Computational Science Research Center, Beijing, (2011).

[25] F. Dubois, Equivalent partial differential equations of a lattice Boltzmann scheme, Comput. Math. Appl., 55 (2008), pp. 1141.

[26] U. Frisch, D. D'Humières, B. Hasslacher, P. Lallemand, Y. Pomeau AND J.-P. RivET, Lattice gas hydrodynamics in two and three dimensions, Complex Syst., 1 (1987), pp. 649.

[27] I. GINZBURG AND D. D'HUMIÈRES, Lattice Boltzmann and analytical modeling of flow processes in anisotropic and heterogeneous stratified aquifers, Adv. Water Res., 30 (2007), pp. 2202.

[28] F. Dubois, P. LALlemAND AND M. M. TEKITEK, Using lattice Boltzmann scheme for anisotropic diffusion, Finite Volumes for Complex Applications V, (2008), pp. 795.

[29] F. Dubois, C.-A. LiN AND M. M. TEKITEK, Anisotropic thermal lattice Boltzmann simulation of 2D natural convection in a square cavity, Comput. Fluids, 124 (2016), pp. 278.

[30] X. Y. HE AND L.-S. LUO, Lattice Boltzmann model for the incompressible Navier-Stokes equation, J. Stat. Phys., 88 (1997), pp. 927.

[31] J. WANG, D. H. WANG, P. LALlEMAND AND L.-S. LUO, Lattice Boltzmann simulations of thermal convective flows in two dimensions, Comput. Math. Appl., 65 (2013), pp. 262.

[32] D. A. MAYNE, A. USMANI AND M. CRAPPER, h-adaptive finite element solution of unsteady thermally driven cavity problem, Int. J. Numer. Methods Heat Fluid Flow, 10 (2000), pp. 598.

[33] C.-H. LiU, K.-H. LIN, H.-C. MAI AND C.-A. LIN, Thermal boundary conditions for thermal Lattice Boltzmann simulations, Comput. Math. Appl., 59 (2010), pp. 2178.

[34] N. C. Markatos AND K. A. Pericleous, Laminar and turbulent natural convection in an enclosed cavity, Int. J. Heat Mass Transf., 27 (1984), pp. 755.

[35] D. CONTRinO, P. LAllemand, P. Asinar AND L.-S. LuO, Lattice-Boltzmann simulations of the thermally driven 2D square cavity at high Rayleigh numbers, J. Comput. Phys., 275 (2014), pp. 257.

[36] S. PAOluCCI AND D. R. ChenOWETH, Transition to chaos in defferentially heated vertical cavity, J. Fluid Mech., 201 (1989), pp. 379. 